\title{
Uso de concentrados de fibrinógeno para el tratamiento de la hipofibrinogenemia adquirida
}

\author{
Ignacio Sarmiento G. ${ }^{1}$, David Torres P. ${ }^{1}$, Tiare de la Puente B. ${ }^{1}$, \\ Mario Portilla F. ${ }^{1}$ y Mauricio González L. ${ }^{1}$
}

\section{Use of Fibrinogen Concentrates for the Treatment of Acquired Hypofibrinogenemia}

Introduction: An adequate bleeding management should include a proper assessment of fibrinogen values and consequent replacement. Traditional sources for this hemostatic element include fresh frozen plasma and cryoprecipitates. Lyophilized human fibrinogen concentrates are a novel therapeutic alternative for the chilean market. Aim: This study aims to describe the clinical course of the first patients in our institution receiving fibrinogen concentrates, included in a goal directed hemostatic management algorithm. Materials and Method: Case series of patients with hypofibrinogenemia secondary to severe perioperative bleeding, in which fibrinogen concentrate was used for fibrinogen replacement. Thromboelastometry was used to define dose regimens. Demographic and surgical variables, complications and follow-up up to 3 months were registered. Results: Fibrinogen concentrate was used in 18 patients. Median age was 40.7 (56.5-63) years, and two thirds of the patients were male. Five patients died. All of the cases required postoperative intensive care. Eight patients underwent cardiac surgery. There was a heterogenic use of blood derived products and lyophilized concentrates, but in all cases its use was guided by thromboelastometry. No patients needed a secondary exploration due to bleeding. Conclusion: The use of human fibrinogen concentrate included in a bleeding management algorithm is a feasible hemostatic resource in the chilean current situation. The clinical impact of this intervention requires further evidence-based evaluation.

Key words: fibrinogen concentrate; bleeding; thromboelastometry.

\section{Resumen}

Introducción: Un adecuado manejo del sangrado debe incluir la correcta valoración y eventual reposición de fibrinógeno. Las fuentes tradicionales de este elemento hemostático incluyen el plasma fresco congelado y los crioprecipitados. Los concentrados liofilizados de fibrinógeno humano (CFH) son una alternativa terapéutica novedosa en el mercado chileno. Objetivo: Este estudio describe el curso clínico de los primeros pacientes en nuestra institución requirentes de $\mathrm{CFH}$, dentro de un algoritmo de reposición hemostática por metas. Materiales y Método: Serie de pacientes con hipofibrinogenemia secundaria a sangrado perioperatorio severo, en los que se utilizó CFH como método de reposición de fibrinógeno. Se utilizó tromboelastometría para definir dosis. Se registraron variables demográficas, operatorias, complicaciones y seguimiento hasta los 3 meses. Resultados: Se utilizaron CFH en 18 pacientes. La mediana de edad fue 40,7 (56,5-63) años y dos tercios de los pacientes fueron de sexo masculino. Fallecieron 5 pacientes de la serie. Todos los pacientes requirieron manejo posoperatorio en una unidad de cuidados intensivos. Ocho pacientes fueron sometidos a cirugía cardiaca. El uso de hemocomponentes y concentrados liofilizados fue heterogéneo, pero en todos los casos su uso fue determinado por tromboelastometría. Ningún paciente fue reintervenido a causa de sangrado posoperatorio. Conclusión: El uso de concentrados de fibrinógeno humano dentro de un algoritmo de manejo de sangrado guiado por tromboelastometría, es un recurso hemostático factible en la realidad nacional. El impacto clínico de esta intervención requiere una subsiguiente evaluación basada en la evidencia.

Palabras clave: concentrado de fibrinógeno; sangrado; tromboelastometría.
'Servicio de Anestesiología, Clínica Santa María. Universidad de Los Andes, Santiago, Chile.

Recibido 2020-10-13, aceptado 2020-12-09

Correspondencia a: David Torres P. dtorres@miuandes.c 


\section{Introducción}

El manejo adecuado y oportuno del sangrado es fundamental en la práctica de la medicina de urgencias. La alteración de la hemostasia secundaria a la pérdida sanguínea excesiva lleva a un ciclo vicioso de coagulopatía, requerimientos transfusionales y morbimortalidad en estos pacientes ${ }^{1}$. Durante el shock hipovolémico por sangrado, el fibrinógeno es el primer componente de la hemostasia en alcanzar valores $\operatorname{críticos}^{2}$. La hipofibrinogenemia (definida como la concentración de fibrinógeno plasmático por debajo de los 1,5-2 g/L) se ha asociado a desenlaces adversos en múltiples escenarios clínicos, tales como trauma, cirugía cardiaca, trasplante hepático, hemorragia postparto y pediatría $a^{3-8}$. El establecimiento de estrategias de manejo del sangrado, que incluyan corrección de la hipofibrinogenemia, ha llevado a una mejoría en los resultados clínicos ${ }^{8-11}$. Por ello, se pregona su corrección ${ }^{8,12,13}$.

Clásicamente las fuentes de fibrinógeno para reposición han sido el plasma fresco congelado y los crioprecipitados, ambos hemocomponentes que requieren de un elaborado proceso de adquisición, almacenaje y administración ${ }^{14,15}$. En los últimos años ha surgido la utilización de concentrados de fibrinógeno humano (CFH) como una alternativa en la corrección de la hipofibrinogenemia adquirida. Estos concentrados están disponibles como productos liofilizados, pasteurizados, derivados de un grupo de donantes de plasma habiendo sido tratados mediante purificación e inactivación viral. Estos no requieren cross match transfusional ni descongelamiento, tienen una concentración estable de fibrinógeno, son fácilmente almacenables a temperatura ambiental y rápidos en su administración ${ }^{15-18}$. Además, están aprobados por organismos internacionales para el manejo de la hipofibrinogenemia adquirida ${ }^{16}$. La evidencia reciente apunta a una no inferioridad en desenlaces clínicos respecto al uso de $\mathrm{CFH}^{19-21}$, además de disminuir los requerimientos transfusionales de glóbulos rojos ${ }^{22}$. Esto cobra importancia considerando la crisis actual de sustentabilidad de los bancos de sangre, en especial a raíz de la pandemia por COVID-1923,24.

Debido a la falta de experiencia en el uso de este producto en Chile, se elaboró en nuestro centro un protocolo de administración de CFH. La importación de CFH liofilizado se logró a través de una resolución excepcional del Ministerio de Salud $^{25}$, a la espera de la aprobación definitiva por parte del Instituto de Salud Pública de la utilización de CFH para el manejo de la hipofibrinogenemia adquirida.
El objetivo de la siguiente serie es exponer el consumo de hemocomponentes en los pacientes que recibieron $\mathrm{CFH}$ en el perioperatorio. Objetivos secundarios son describir las características clínicas, resultados y factibilidad de su uso en la realidad local, tras haber aplicado este protocolo.

\section{Materiales y Método}

Se presenta una serie de casos de pacientes quirúrgicos con hipofibrinogenemia adquirida por sangrado en los cuales, dentro de su terapia hemostática, se utilizó CFH (Fibryga ${ }^{\circledR}$, Octapharma, Laachen, Suiza) como método de reposición de fibrinógeno. La adquisición y revisión de datos clínicos de los pacientes fue autorizado por el comité ético-científico local.

\section{Pacientes}

Pacientes con hipofibrinogenemia secundaria a sangrado severo perioperatorio (definido como sangrado estimado mayor al $20 \%$ de la volemia, o bien que produjera shock hipovolémico, o en un órgano crítico) y que recibieron CFH como parte de su manejo ${ }^{8}$. En una hoja de recopilación de datos se registraron variables demográficas, operatorias, complicaciones y seguimiento hasta los 3 meses.

\section{Determinación del fibrinógeno plasmático y tromboelastometría}

Para identificar aquellos pacientes con hipofibrinogenemia ${ }^{8}$, se utilizó la tromboelastometría $(R O$ TEM Sigma, Instrumental Laboratories, Bedford, MA USA) extrayendo una muestra de sangre total recolectada en un tubo de $2,7 \mathrm{ml}$ con citrato de sodio al 3,2\% (BD Vacutainer, Franklin Lakes NJ, USA). Esta tecnología permite la adquisición de valores tanto en ensayos específicos de polimerización de fibrina (FIBTEM), como en pruebas generales de la coagulación tanto de la vía extrínseca (EXTEM) como de la vía intrínseca (INTEM) de manera simultánea. La medición concomitante de la concentración plasmática de fibrinógeno por método de Clauss no fue realizada de manera sistemática.

\section{Protocolo de reposición}

La estrategia de reposición de fibrinógeno se definió a partir de la elaboración de un algoritmo de manejo del sangrado severo guiado por tromboelastometría (Figura 1), en donde la reposición de fibrinógeno se realiza conforme a los resultados de la amplitud en milímetros de la prueba de polimerización de fibrina FIBTEM ${ }^{15,26}$ : 
Dosis Fibrinógeno $(g)=($ FIBTEM MCF deseado (mm) - FIBTEM MCF actual $(\mathrm{mm})) x$ (peso corporal (kg)/140)

Para facilitar la aplicación del algoritmo se decidió establecer dosificaciones fijas de fibrinógeno en relación con los hallazgos de las pruebas de tromboelastometría específicas para polimerización de fibrina (FIBTEM), como lo detalla la Figura 1.

Nuestro algoritmo transfusional se elaboró en base a una extensa revisión de la literatura ${ }^{27}$, considerando riesgos y beneficios de cada umbral de reposición y buscando priorizar la utilización de concentrados liofilizados tanto de fibrinógeno como de complejos protrombínicos.

Respecto a la administración de estos concen- trados liofilizados, los viales fueron reconstituidos y administrados según las especificaciones del proveedor.

\section{Análisis estadístico}

Los hallazgos se presentan de manera descriptiva. Las variables se presentan como mediana (rango intercuartil) o como números absolutos.

\section{Resultados}

Se presenta una serie de 18 pacientes que recibieron concentrados de fibrinógeno humano liofilizado $(\mathrm{CFH})$ en nuestro servicio de pabellón central entre

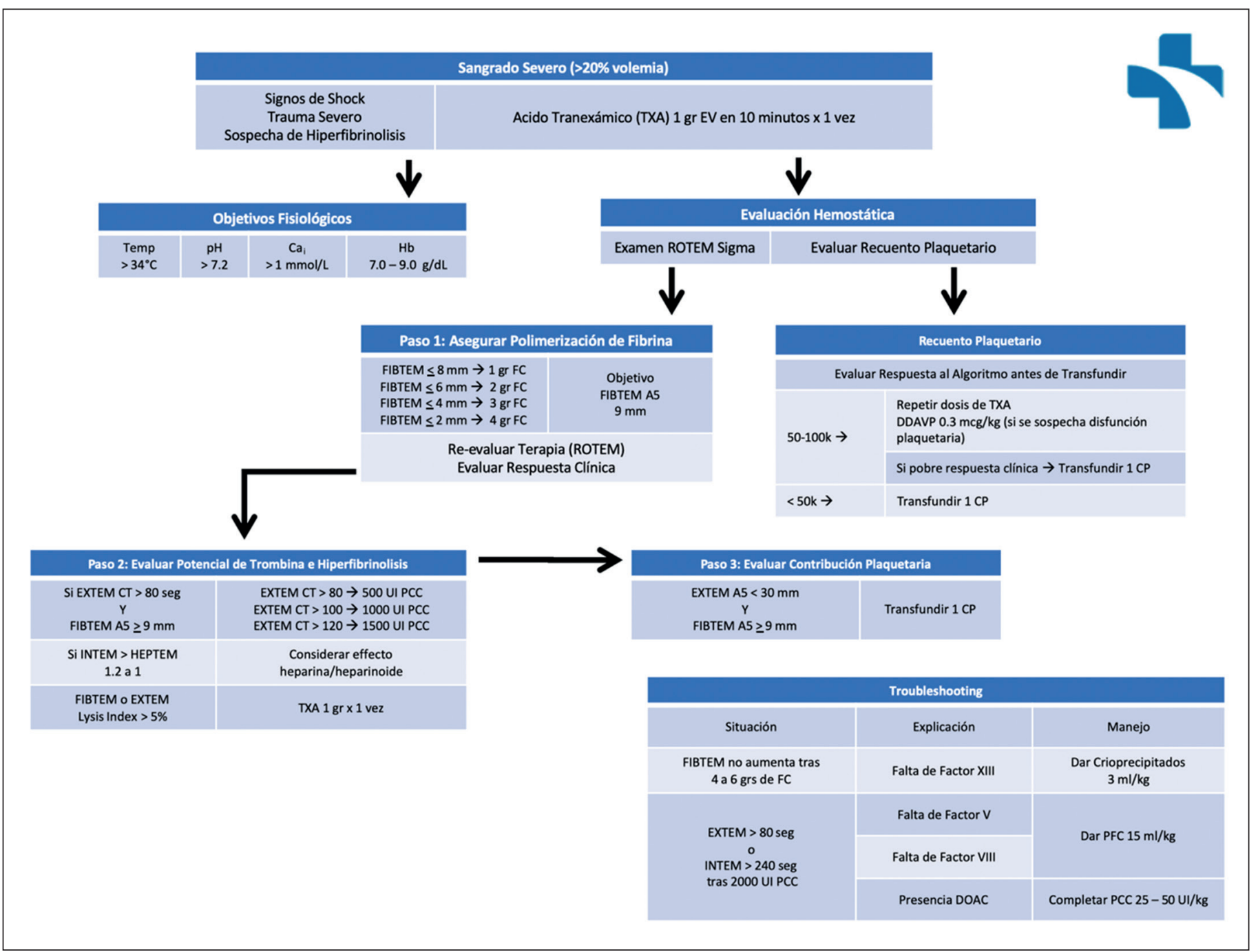

Figura 1. Algoritmo de manejo del sangrado severo mediante tromboelastometría ROTEM. EV, Endovenoso. Temp, temperatura. Ca., calcio iónico. Hb, hemoglobina. Mm, milímetros. G, gramos. A5, amplitud en milímetros de la prueba a los 5 minutos. CT, tiempo de coagulación. Seg, segundos. PCC, complejo protrombínico. TXA, ácido tranexámico. DDAVP, desmopresina. CP, concentrado de plaquetas. DOAC, anticoagulantes directos no vitamina $\mathrm{K}$ dependientes. 


\section{ARTíCULO ORIGINAL}

Tabla 1. Características clínicas de los pacientes que recibieron concentrados liofilizados de fibrinógeno humano (CFH)

\begin{tabular}{|lll|}
\hline Variable & & $\begin{array}{l}\text { Mediana (rango intercuartil) o } \\
\text { valor absoluto }\end{array}$ \\
Edad & Masculino & $40,7(56,5-63)$ \\
Género & 12 \\
Índice masa corporal & & $24,4(21,5-29,6)$ \\
Comorbilidades & Tabaquismo & 9 \\
& HTA & 9 \\
& Obesidad (IMC > 30) & 4 \\
& Arritmias & 4 \\
& Dislipidemia & 2 \\
& DM & 2 \\
& Ateroesclerosis & 1 \\
& Cardiopatía coronaria & 1 \\
& Uso AAP & 1 \\
& Uso TACO & 1 \\
Tipo de cirugía & Cardiaca & 8 \\
& No cardiaca & 10 \\
\hline
\end{tabular}

IMC: Índice de masa corporal. HTA: Hipertensión arterial. DM: Diabetes mellitus. AAP: antiagregación plaquetaria. TACO: terapia anticoagulante oral.

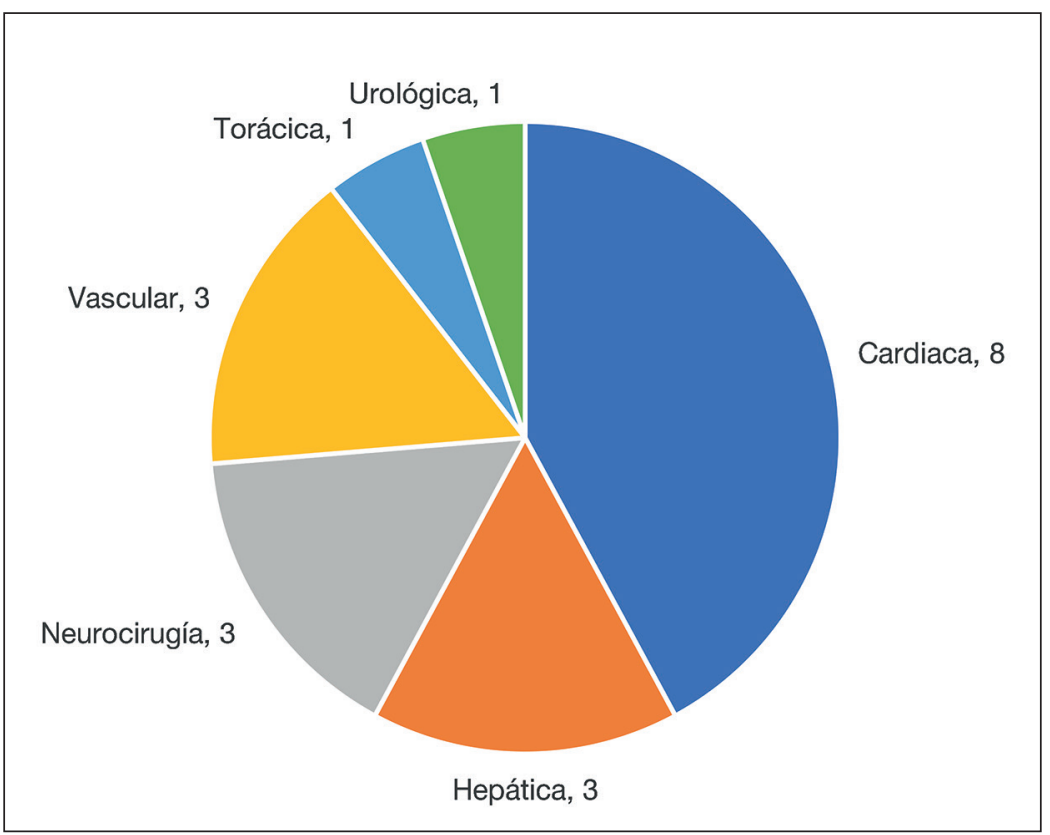

Figura 2. Cirugías en las que se utilizaron concentrados liofilizados de fibrinógeno humano (CFH). Tipo de cirugía, frecuencia. el 1 de mayo de 2019 y el 31 de enero de 2020, como parte de un protocolo reanimación hemostática basado en tests viscoelásticos. Las características clínicas de los pacientes se presentan en la Tabla 1. Destaca una mediana de años de edad de 40,7 (56,5$63)$ y que dos tercios de los pacientes eran de sexo masculino. Ocho de las cirugías correspondieron a cirugía cardiaca, el detalle de las restantes se explicita en el Figura 2.

En todos los pacientes se logró dosificar la administración de $\mathrm{CFH}$ mediante trombelastometría ROTEM. Ninguna muestra extraída debió ser descartada ni ningún examen arrojó errores de procesamiento. En los 18 pacientes, la administración de CFH se realizó después de contar con los resultados de la prueba FIBTEM de tromboelastometría. Mediante esta tecnología se objetivó la necesidad de reponer fibrinógeno y se dosificó en relación con los hallazgos de la prueba FIBTEM y el uso del algoritmo detallado en la Figura 1.

La dosis de reposición con CFH fue de 2 (2-3) gramos, lo que corresponde a 36 (27-58) gramos por kilo de peso. Destaca que dos tercios de los pacientes requirieron al menos una unidad de glóbulos rojos, con una mediana de $2(0-6)$ unidades por paciente. El requerimiento de hemocomponentes y productos hemostáticos en las primeras 24 horas perioperatorias se desglosa en la Tabla 2 . Tres pacientes no requirieron hemocomponentes o productos hemostáticos más allá del CFH. Solo un paciente de esta serie requirió una transfusión masiva, definida como la necesidad de una transfusión mayor a 10 unidades de glóbulos rojos en $24 \mathrm{~h}^{28}$.

La mortalidad general, a 28 y a 90 días, fue de 5 de 18 pacientes (Figura 3 ) siendo todos los fallecimientos intrahospitalarios. Todos los individuos de la serie realizaron su posoperatorio en una Unidad de Paciente Crítico (UCP). La estadía en UPC fue de 3 (1-4) días y la estadía hospitalaria total de 12 (7-19) días.

Siete pacientes presentaron sangrado mayor posoperatorio según criterios de la International Society of Thrombosis and Hemostasis (ISTH) ${ }^{29}$. Sin embargo, ningún paciente fue reexplorado a causa de este motivo. Dos pacientes requirieron revisiones quirúrgicas; el primero, presentó una colección abscedada en el sitio operatorio, mientras que el segundo correspondió a una embolización angiográfica del remanente de una malformación arteriovenosa gigante, no resuelta completamente en el primer tiempo quirúrgico.

Once de los 18 pacientes permanecieron en ventilación mecánica una vez terminada la cirugía, con $1(0-1)$ día de permanencia en soporte ventilatorio invasivo. 
Tabla 2. Uso de hemocomponentes y productos hemostáticos en las primeras 24 horas perioperatorias

\begin{tabular}{|lccc|}
\hline Tipo de componente hemostático & Mediana & Rango intercuartil & $\begin{array}{c}\text { Pacientes que requirieron } \\
\text { el componente hemostático }\end{array}$ \\
\hline Glóbulos rojos (U) & 2 & $0-6$ & 11 \\
Concentrados de plaquetas (U) & 0 & $0-1$ & 5 \\
Plasma fresco congelado (U) & 0 & $0-3$ & 6 \\
Crioprecipitados (U) & 0 & $0-5$ & 6 \\
CCP (vial 500 UI fIX) & 2 & $0-3$ & 10 \\
\hline
\end{tabular}

U: unidades. CCP: concentrado de complejos protrombínicos. UI: unidades internacionales. FIX: factor nueve de la coagulación.

Ocho pacientes presentaron injuria renal aguda posoperatoria definida según criterios KDIGO. De estos, 3 pacientes requirieron terapia de reemplazo renal posterior a la cirugía. Tres pacientes presentaron infecciones en el posoperatorio, solo una de ellas fue de sitio quirúrgico.

\section{Discusión}

En este estudio describimos la primera experiencia chilena del uso de concentrados de fibrinógeno humano liofilizado como parte del manejo de la hipofibrinogenemia adquirida durante el sangrado severo perioperatorio, enfatizando que esta es una terapia factible para nuestra realidad nacional.

Cinco de los 18 pacientes fallecieron. Estos resultados se asemejan a los obtenidos en series mayores destinadas a la evaluación de algoritmos de manejo del trauma grave mediante métodos viscoelásti$\cos ^{10}$, lo que refleja la gravedad de los pacientes de la serie. La estadía en UPC fue de 3 (1-4) días, con una estadía hospitalaria total de 12 (7-19) días. Estos resultados son comparables a lo descrito en la literatura, donde se estudió dirigidamente sangrado perioperatorio o reposición de fibrinógeno $0^{1,20}$.

El consumo de hemocomponentes en las primeras 24 horas perioperatorias no fue homogéneo en la serie. La naturaleza de las cirugías que requirieron un manejo activo del sangrado es similar a la descrita en otras series ${ }^{30,31}$. En línea con el algoritmo utilizado (Figura 1), resaltan el uso de unidades de glóbulos rojos para mantener una adecuada entrega periférica de oxígeno, y la utilización de concentrados protrombínicos para optimizar la generación de trombina, manteniendo la preponderancia de la reposición de fibrinógeno como pilar inicial de la terapia hemostática ${ }^{2,8}$.

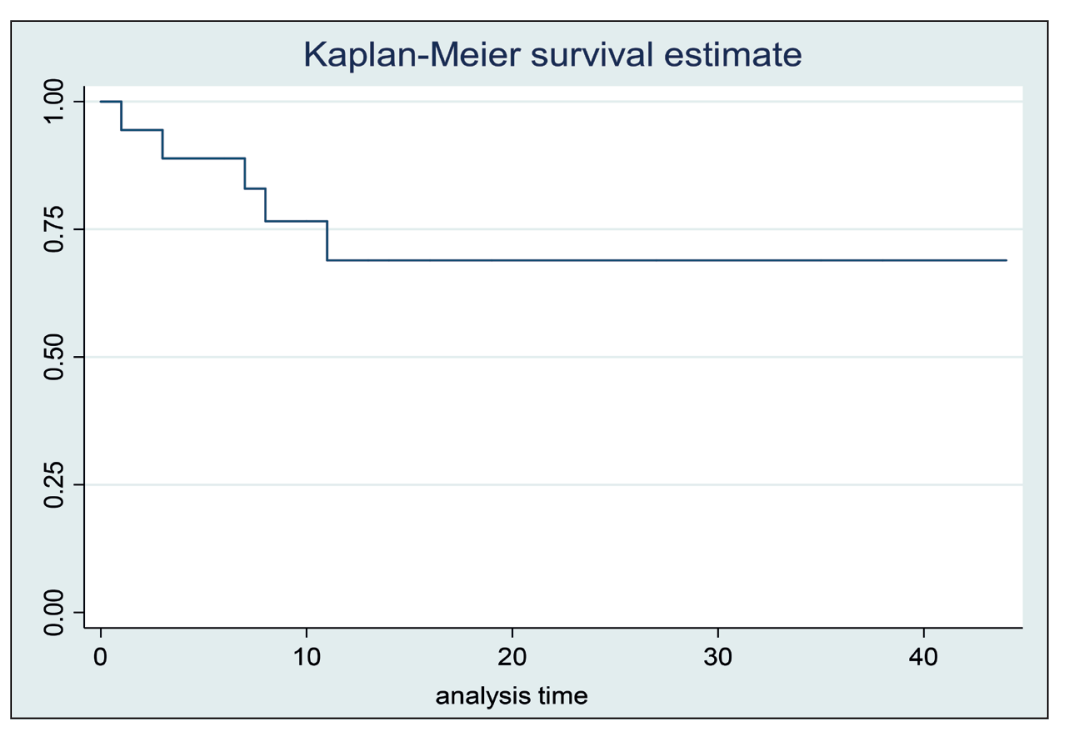

Figura 3. Proyección de mortalidad general según análisis de Kaplan-Meier.

La elaboración del algoritmo de manejo del sangrado se realizó de manera de disminuir la exposición del paciente a hemocomponentes ${ }^{27}$. Esta forma de optimización de la coagulación es parte de una estrategia multidisciplinaria conocida como Patient Blood Management, y que busca optimizar la masa eritrocitaria propia del paciente, racionalizando la terapia transfusional, en aras de mejorar los resultados clínicos ${ }^{32,33}$.

La pandemia de COVID-19 ha desenmascarado la necesidad de contar con alternativas más costoefectivas y sustentables a los hemocomponentes ${ }^{23,33}$. Una terapia transfusional juiciosa basada en los pilares del Patient Blood Management ha demostrado ser sustentable en el tiempo y rentable para las instituciones de salud que la implementan ${ }^{34}$. 
Existe consenso que un algoritmo de manejo del sangrado basado en el uso de concentrados liofilizados es una alternativa efectiva a la terapia habitual basada en reposición hemostática mediante hemocomponentes ${ }^{35,36}$. La reposición de fibrinógeno mediante CHF ha resultado al menos no inferior a la realizada con crioprecipitado respecto a resultados clínicos y seguridad ${ }^{20-22}$, pero significativamente más veloz en su administración ${ }^{18}$. Nuestro estudio no buscó dirigidamente replicar estos resultados, pero la aplicabilidad del algoritmo de manejo utilizando ROTEM y CHF permite consolidar las bases para el establecimiento de protocolos de investigación en esta área de trabajo en la realidad local.

La reposición de fibrinógeno, independientemente de la fuente que se utilice, debiera guiarse por metas para obtener resultados óptimos y consistentes ${ }^{21,22}$. La rápida capacidad de determinar la dosis adecuada de fibrinógeno para optimizar la polimerización de fibrina en estos pacientes mediante tromboelastometría está bien documentada ${ }^{37-39}$.

Este trabajo presenta limitaciones; es una serie pequeña, sin grupo control y con una clara heterogeneidad respecto a las características clínicas que llevaron a la necesidad de reposición de fibrinógeno. Por el carácter descriptivo de los datos, se hace dificultoso realizar inferencias respecto a la efectividad o seguridad de esta novedosa estrategia terapéutica. Esta serie permite, sin embargo, catalogar como plausible la aplicabilidad del algoritmo propuesto.
La adquisición de los implementos técnicos, la elaboración y revisión de protocolos de manejo y la capacitación del recurso humano en el uso de estas nuevas tecnologías, son fundamentales para la sustentabilidad de esta terapia.

Este es la primera descripción del uso de concentrados liofilizados de fibrinógeno humano en pacientes chilenos. Se necesitan más estudios para evaluar la efectividad de esta intervención, comparándolo con el estado de cuidado actual. La literatura internacional sugiere un beneficio en incorporar esta herramienta dentro del arsenal hemostático y reconocemos como importante la integración de esta terapia en la realidad nacional.

\section{Responsabilidades éticas}

Protección de personas y animales. Los autores declaran que para esta investigación no se han realizado experimentos en seres humanos ni en animales.

Confidencialidad de los datos. Los autores declaran que en este artículo no aparecen datos de pacientes.

La adquisición y revisión de datos clínicos de los pacientes fue autorizada por el comité éticocientífico local.

Conflictos de interés: no hay.

\section{Bibliografía}

1. Ranucci M, Baryshnikova E, Castelvecchio S, Pelissero G. Surgical, Clinical Outcome Research G. Major bleeding, transfusions, and anemia: the deadly triad of cardiac surgery. Ann Thorac Surg. 2013;96:478-85.

2. Hiippala ST, Myllyla GJ, Vahtera EM Hemostatic factors and replacement of major blood loss with plasma-poor red cell concentrates. Anesth Analg. 1995;81:360-5.

3. Charbit B, Mandelbrot L, Samain E, Baron G, Haddaoui B, Keita H, et al. The decrease of fibrinogen is an early predictor of the severity of postpartum hemorrhage. J Thromb Haemost. 2007;5:266-73.

4. Rourke C, Curry N, Khan S, Taylor R, Raza I, Davenport R, et al. Fibrinogen levels during trauma hemorrhage, response to replacement therapy, and association with patient outcomes. J Thromb Haemost. 2012;10:1342-51.

5. Karkouti K, Callum J, Crowther MA, McCluskey SA, Pendergrast J, Tait G, et al. The relationship between fibrinogen levels after cardiopulmonary bypass and large volume red cell transfusion in cardiac surgery: an observational study. Anesth Analg. 2013;117:14-22.

6. Hagemo JS, Stanworth S, Juffermans NP, Brohi K, Cohen M, Johansson PI, et al. Prevalence, predictors and outcome of hypofibrinogenaemia in trauma: a multicentre observational study. Crit Care. 2014;18:R52.

7. Haas T, Spielmann N, Restin T, Seifert B, Henze G, Obwegeser J, et al. Higher fibrinogen concentrations for reduction of transfusion requirements during major paediatric surgery: A prospective randomised controlled trial. Br J Anaesth. 2015;115:234-43.
8. Kozek-Langenecker SA, Ahmed AB, Afshari A, Albaladejo P, Aldecoa C, Barauskas G, et al. Management of severe perioperative bleeding: guidelines from the European Society of Anaesthesiology: First update 2016. Eur J Anaesthesiol. 2017;34:332-95.

9. Nardi G, Agostini V, Rondinelli B, Russo E, Bastianini B, Bini G, et al. Trauma-induced coagulopathy: impact of the early coagulation support protocol on blood product consumption, mortality and costs. Crit Care 2015;19:83.

10. González E, Moore EE, Moore HB, Chapman MP, Chin TL, Ghasabyan A, et al. Goal-directed Hemostatic Resuscitation of Trauma-induced Coagulopathy: A Pragmatic Randomized Clinical Trial Comparing a Viscoelastic Assay to Conventional Coagulation Assays. Ann Surg. 2016;263:1051-9. 
11. Snegovskikh D, Souza D, Walton Z, Dai F, Rachler R, Garay A, et al. Point-of-care viscoelastic testing improves the outcome of pregnancies complicated by severe postpartum hemorrhage. J Clin Anesth. 2018;44:50-6.

12. Erdoes G, Koster A, Meesters MI, Ortmann E, Bolliger D, Baryshnikova $\mathrm{E}$, et al. The role of fibrinogen and fibrinogen concentrate in cardiac surgery: an international consensus statement from the Haemostasis and Transfusion Scientific Subcommittee of the European Association of Cardiothoracic Anaesthesiology. Anaesthesia 2019;74:1589-600.

13. Spahn DR, Bouillon B, Cerny V, Duranteau J, Filipescu D, Hunt BJ, et al. The European guideline on management of major bleeding and coagulopathy following trauma: fifth edition. Crit Care 2019;23:98.

14. Idris SF, Hadjinicolaou AV, Sweeney M, Winthrop C, Balendran G, Besser M. The efficacy and safety of cryoprecipitate in the treatment of acquired hypofibrinogenaemia. Br J Haematol. 2014;166:458-61.

15. Levy JH, Goodnough LT. How I use fibrinogen replacement therapy in acquired bleeding. Blood 2015;125:138793.

16. European Medicines Agency Science Medicines Health. Guideline on core SmPC for human fibrinogen products 2015 [Available from: https://www. ema.europa.eu/en/documents/scientificguideline/guideline-core-summaryproduct-characteristics-smpcfor-humanfibrinogen-products_en.pdf.

17. Costa-Filho R, Hochleitner G, Wendt M, Teruya A, Spahn DR. Over 50 Years of Fibrinogen Concentrate. Clin Appl Thromb Hemost. 2016;22:109-14.

18. Roy A, Stanford S, Nunn S, Alves S, Sargant N, Rangarajan S, et al. Efficacy of fibrinogen concentrate in major abdominal surgery - A prospective, randomized, controlled study in cytoreductive surgery for pseudomyxoma peritonei. J Thromb Haemost. 2020;18:352-63.

19. Grottke O, Schochl H, Levy JH, Hunt BJ. Supplementary fibrinogen in the management of bleeding: re-evaluation of data from clinical trials. Br J Anaesth. 2018;120:407-9.
20. Callum J, Farkouh ME, Scales DC, Heddle NM, Crowther M, Rao V, et al. Effect of Fibrinogen Concentrate vs Cryoprecipitate on Blood Component Transfusion After Cardiac Surgery: The FIBRES Randomized Clinical Trial. JAMA 2019:1-11.

21. Cushing MM, Haas T. Fibrinogen concentrate for perioperative bleeding: what can we learn from the clinical trials? Transfusion 2019;59:3295-7.

22. Fabes J, Brunskill SJ, Curry N, Doree C, Stanworth SJ. Pro-coagulant haemostatic factors for the prevention and treatment of bleeding in people without haemophilia. Cochrane Database Syst Rev. 2018;12:CD010649.

23. Klein HG, Hrouda JC, Epstein JS. Crisis in the Sustainability of the U.S. Blood System. N Engl J Med. 2017;377:1485-8.

24. Wang Y, Han W, Pan L, Wang C, Liu $\mathrm{Y}, \mathrm{Hu} \mathrm{W}$, et al. Impact of COVID-19 on blood centres in Zhejiang province China. Vox Sang. 2020.

25. Mora J. Resolución exenta 1514: Autorízase a Laboratorio Bagó de Chile S.A., para importar según artículo ${ }^{\circ} 99$ del Código Sanitario, decreto con fuerza de ley $n^{\circ} 725$, respecto del producto farmacéutico fibryga liofilizado para solución inyectable/perfusión. In: Instituto de Salud Pública de Chile, editor. 2019.

26. Tanaka KA, Esper S, Bolliger D. Perioperative factor concentrate therapy. Br J Anaesth. 2013;111 Suppl 1:i35-49.

27. Gorlinger K, Perez-Ferrer A, Dirkmann D, Saner F, Maegele M, Calatayud AAP, et al. The role of evidence-based algorithms for rotational thromboelastometryguided bleeding management. Korean J Anesthesiol. 2019;72:297-322.

28. Mesar T, Larentzakis A, Dzik W, Chang Y, Velmahos G, Yeh DD. Association Between Ratio of Fresh Frozen Plasma to Red Blood Cells During Massive Transfusion and Survival Among Patients Without Traumatic Injury. JAMA Surg. 2017;152:574-80.

29. Schulman S, Angeras U, Bergqvist D, Eriksson B, Lassen MR, Fisher W, et al. Definition of major bleeding in clinical investigations of antihemostatic medicinal products in surgical patients. J Thromb Haemost. 2010;8:202-4.

30. Irita K. Risk and crisis management in intraoperative hemorrhage: Human factors in hemorrhagic critical events. Korean J Anesthesiol. 2011;60:151-60.

31. Althoff FC, Neb H, Herrmann E, Trentino KM, Vernich L, Fullenbach C, et al. Multimodal Patient Blood Management Program Based on a Three-pillar Strategy: A Systematic Review and Meta-analysis. Ann Surg. 2019;269:794-804.

32. Meybohm P, Herrmann E, Steinbicker AU, Wittmann M, Gruenewald M, Fischer D, et al. Patient Blood Management is Associated With a Substantial Reduction of Red Blood Cell Utilization and Safe for Patient's Outcome: A Prospective, Multicenter Cohort Study With a Noninferiority Design. Ann Surg. 2016;264:203-11.

33. Shander A, Goobie SM, Warner MA, Aapro M, Bisbe E, Perez-Calatayud AA, et al. Essential Role of Patient Blood Management in a Pandemic: A Call for Action. Anesth Analg. 2020;131:74-85.

34. Kaserer A, Rossler J, Braun J, Farokhzad F, Pape HC, Dutkowski P, et al. Impact of a Patient Blood Management monitoring and feedback programme on allogeneic blood transfusions and related costs. Anaesthesia 2019;74:1534-41.

35. Franchini M, Lippi G. Fibrinogen replacement therapy: a critical review of the literature. Blood Transfus. 2012;10:237.

36. Innerhofer $\mathrm{P}$, Fries D, Mittermayr M, Innerhofer $\mathrm{N}$, von Langen $\mathrm{D}$, Hell $\mathrm{T}$, et al. Reversal of trauma-induced coagulopathy using first-line coagulation factor concentrates or fresh frozen plasma (RETIC): a single-centre, parallel-group, open-label, randomised trial. Lancet Haematol. 2017;4:e258-e71.

37. Schochl H, Schlimp CJ. Trauma bleeding management: the concept of goaldirected primary care. Anesth Analg. 2014;119:1064-73.

38. Pruller F, Munch A, Preininger A, Raggam RB, Grinschgl Y, Krumnikl J, et al. Comparison of functional fibrinogen $(\mathrm{FF} /$ CFF) and FIBTEM in surgical patients - a retrospective study. Clin Chem Lab Med. 2016;54:453-8.

39. Mileo FG, Kataife ED, Adrover A, Garcia Fornari G, Lopez MS, Martinuzzo ME. The haemostasis traffic light: a pragmatic tool for bleeding management. Anaesthesia. 2019;74:1623-4. 